\title{
Fabrication and installation of moderate size shock tube facility - a case study
}

\begin{abstract}
A moderate size shock tube of $7 \mathrm{~m}$ long with inner diameter of $55 \mathrm{~mm}$ and thickness of $20 \mathrm{~mm}$ has been is designed in-house and installed successfully in the Mechanical Engineering Department at Indian Institute of Technology Guwahati (IITG). The shock wave is generatedby rupturing metallic diaphragms instantly with appropriate pressure ratios across the driver $(2 \mathrm{~m})$ and driven $(5 \mathrm{~m})$ section of the shock tube. The pressure rise across the across primary and reflected shock is measured by high frequency pressure transducers located towards end of driven section. All necessary shock tube parameters are calculated through one-dimensional shock tube relations and are validated with experimental data.
\end{abstract}

Volume I Issue I - 2017

Sumit Agarwal, NiranjanSahoo

Department of Mechanical Engineering, Indian Institute of

Technology Guwahati, India

Correspondence: Niranjan Sahoo, Department of Mechanical Engineering, Indian Institute of Technology Guwahati, India, E-mail shock@iitg.ernet.in

Received: May 07, 2017 | Published: May 07, 2017

Keywords: shock tube, shock mach number, primary shock, reflected shock, pressure ratio

\section{Introduction}

Missiles and launch vehicles experience various flow regimes in their flight path. Design of these high speed configurations depend mainly on flow and flight parameters. The more realistic approach to understand the high speed flow physics would be to simulate the high speed flow conditions in the laboratories. During the 1960's considerable research was conducted to establish ground test facilities to simulate such high speed flows. Mainly, the research was focused to understand the aerodynamic data for simple geometries such as cones/ slender bodies. Many analytical models were developed to validate the data for understanding aerodynamic behavior. ${ }^{1,2}$ But, due to lack of availability of high speed sensors and flow diagnostic instrumentation, these facilities provided limited data and could not be initiated for further developments. With advent of computational power, the new era relies heavily on computational codes to simulate flow parameters for complex aerodynamic body shapes. Again, validation of these codes against experimental test bench plays a vital role to understand flow physics and future development of numerical code. So, groundbased experimentation in high-speed impulse test facilities remains an important component for future developments. In such facilities, the aerodynamic testing is considered as highly specialized activity because short duration flows are produced with test times in the range of few milliseconds. They include small and large scale developments in shock tunnels, free-piston shock tunnel and expansion tubes etc., established over last few decades. ${ }^{3-7}$ Main attraction of short duration facilities is that they provide a high-enthalpy slug of test gas at reasonable cost. The test gas is compressed and subsequently expanded with appropriate nozzle to achieve desired conditions in the test section of the tunnel. Though, there are difficulties in the process of data acquisition in the short test times, but the modern days of highspeed instrumentation and flow diagnostics techniques have produced ingenious solutions to take the advantage of short test times.

Shock tube is one of the simple laboratory tools that can generate various flow speeds at different flow regimes. ${ }^{8-9}$ However, the penalty paid for these experimental flow field simulations is the test time which is in the order of milliseconds. It leads to sophisticated instruments to gather all experimental information in a short time scale which is quite challenging. ${ }^{10-11}$ Extending this facility to shock tunnels, researchers conducted force measurement successfully on a missile shaped configuration at supersonic and hypersonic Mach numbers..$^{12-13}$ The surface heating measurements on blunt bodies for variety of interdisciplinary space science applications have also been exhaustively carried out. ${ }^{14-16}$

With the above viewpoint, the authors report about installation of a particular shock tube facility as one of the case study. Being, indigenously designed and fabricated in-house, this modular shock tube facility is very unique in the north-eastern region of the country (India). Apart from aerodynamic experiments at low supersonic speeds, this modular shock tube can also be useful for chemical kinetic studies and other interdisciplinary applications of shock waves. Considering the geographical location of IITG, the major milestone of this facility development are the in-house designed and fabrication of shock tube with allied hardware and integrated effort of the instrumentation for high-speed data acquisition.

\section{Shock tube performance-sample results and discussions}

The shock tube performance is based entirely upon the pressure ratios and speeds of sound in driver and driven section. Since, the strength of the shock increases with increase in the ratio of speeds of sound, it is desirable to have a driver gas with a low molecular weight and driven gas with a high molecular weight. So, the strongest shock wave can be obtained by using a heavy driven gas and a light driver gas. While meeting these requirements, the present investigation is aimed for two driver gases (nitrogen and helium) with air in the driven section and the fundamental one-dimensional relations are used to find the performance parameters of the shock tube. ${ }^{17}$ The complete schematic overviews along with the components of shock tube are shown in (Figure 1). Initially, an aluminum diaphragm separates the driver and driven section of the shock tube. At the beginning of experiment, the pressure inside the driven section (region 1) is maintained at $0.18 \mathrm{bar}$ and all the valves are closed. The driver section is filled with nitrogen through a high pressure cylinder (region 4) and the diaphragm ruptures at a pressure of 20bars. The sudden rupture 
of diaphragm due to pressure difference across the drive and driven section of the tube creates a shock wave that propagates into the driven section. The gas flow starts as a jet and increases as the diaphragm opens, until the cross section of the tube is filled and the shock wave being formed in this process. The sudden rise in pressures across the shock wave induces mass motion in of the driven gas (region 2). The primary shock gets reflected from the end plate, thus forming the reflected shock propagating into a medium which is already at elevated pressure and thereby forming a further increase in pressure and temperature of driven gas (region 5). The pressure jumps across the primary as well as reflected shock are captured from the pressure transducers (Figure 2). From this pressure signals, the time taken by the shock wave to travel a distance of $500 \mathrm{~mm}$ (between pressure transducers) is obtained and subsequently, the shock Mach number $M_{\mathrm{s}}$ is calculated. Further, the analytical expressions (Eq-1) are used to compute the pressure and temperature ratios across primary and reflected shocks. ${ }^{17}$ the results plotted in (Fig-3) have shown very good prediction within the experimental uncertainty of $\pm 10 \%$.

$$
\begin{aligned}
& \frac{p_{2}}{p_{1}}=1+\frac{2 \gamma_{1}}{\gamma_{1}+1}\left(M_{S}^{2}-1\right) ; \frac{p_{5}}{p_{1}}=\left[\frac{2 \gamma_{1} M_{S}^{2}-\left(\gamma_{1}-1\right)}{\left(\gamma_{1}+1\right)}\right]\left[\frac{-2\left(\gamma_{1}-1\right)+M_{S}^{2}\left(3 \gamma_{1}-1\right)}{2+M_{S}^{2}\left(\gamma_{1}-1\right)}\right] \\
& \frac{T_{2}}{T_{1}}=\frac{1+\left(\frac{\gamma_{1}-1}{\gamma_{1}+1}\right) \frac{p_{2}}{p_{1}}}{1+\left(\frac{\gamma_{1}-1}{\gamma_{1}+1}\right) \frac{p_{1}}{p_{2}}} ; \frac{T_{5}}{T_{1}}=\left(\frac{\left[2\left(\gamma_{1}-1\right) M_{S}^{2}+3-\gamma_{1}\right]\left[\left(3 \gamma_{1}-1\right) M_{S}^{2}-2\left(\gamma_{1}-1\right)\right]}{\left(\gamma_{1}+1\right)^{2} M_{s}^{2}}\right)
\end{aligned}
$$

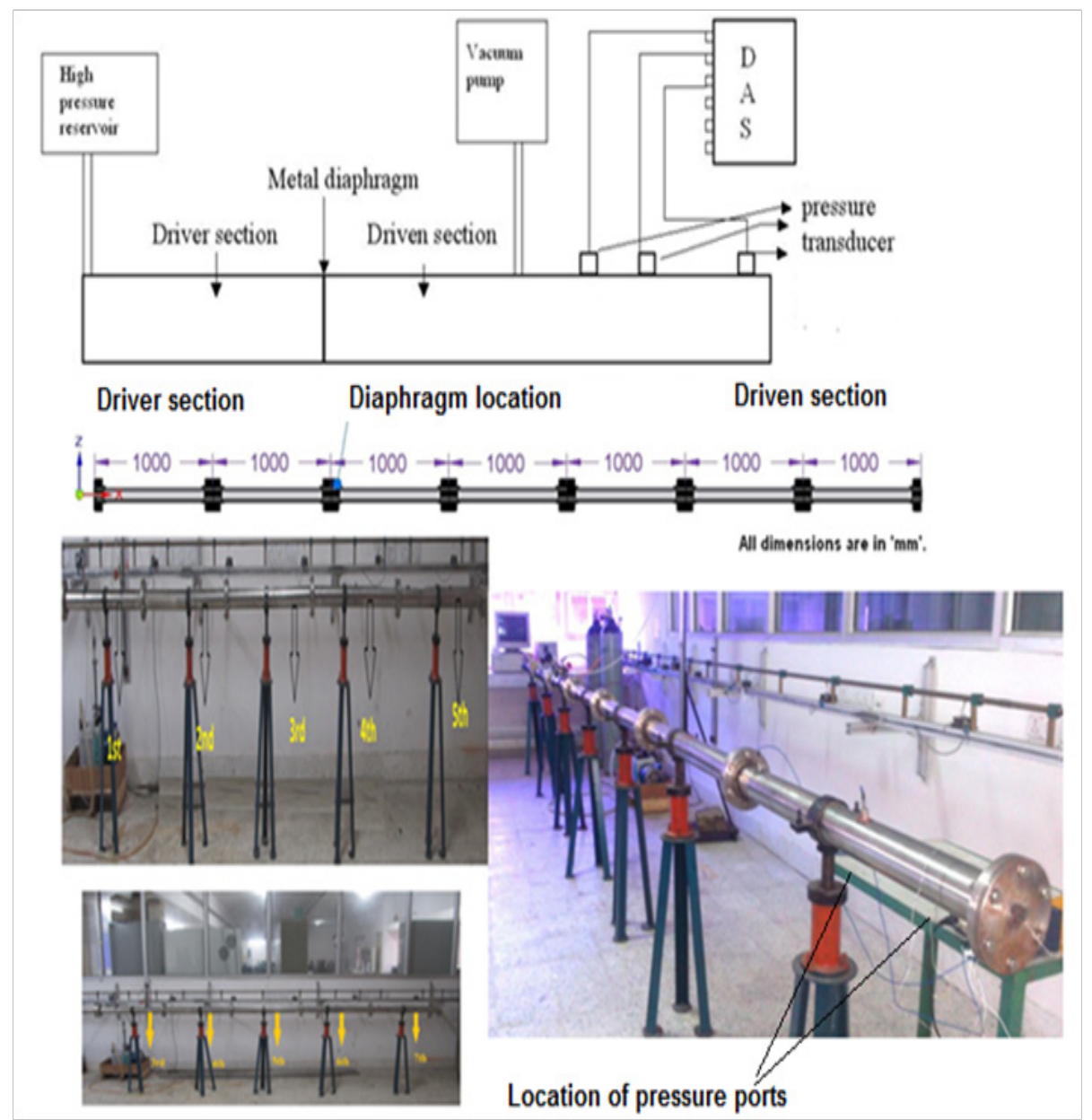

Figure I The modular shock tube facility at IIT Guwahati 


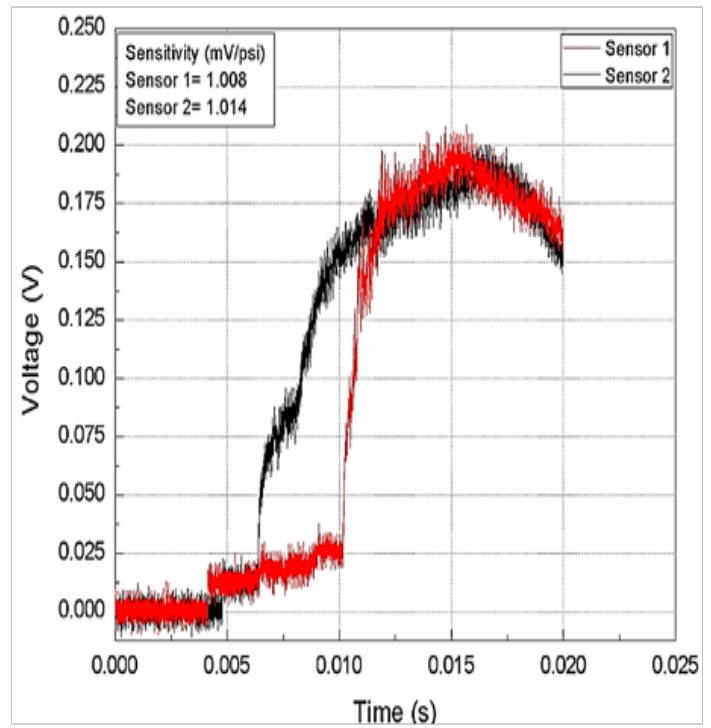

Figure 2 A typical pressure signal from the pressure transducer mounted at driven section of shock tube.

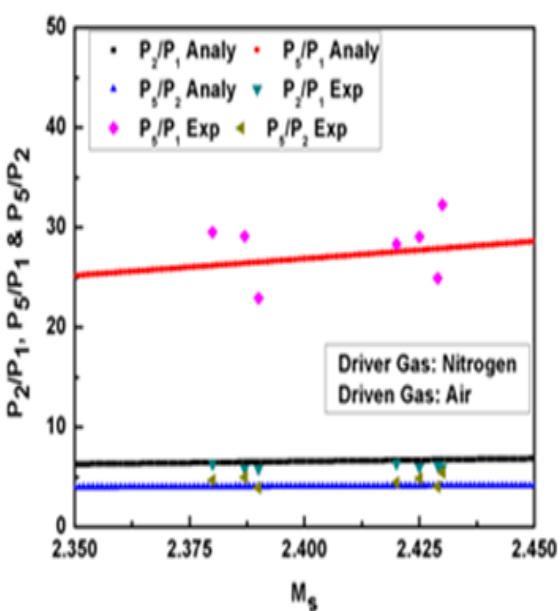

(a)

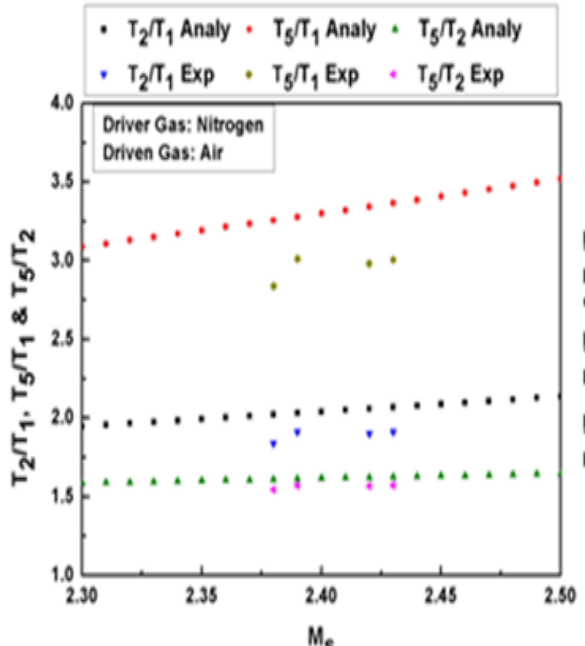

(c)

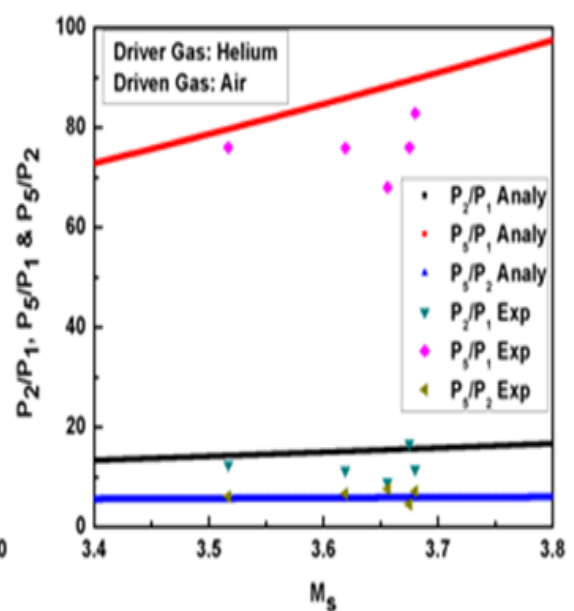

(b)

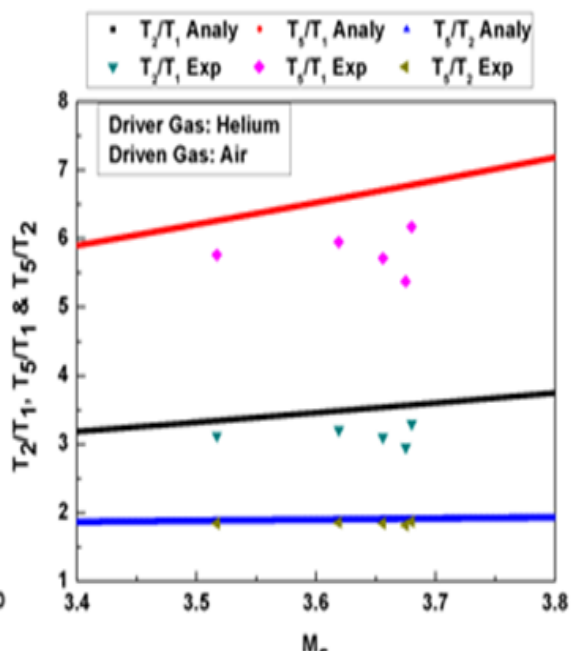

(d)

Figure 3 Comparison of pressure and temperature ratios across primary and reflected shocks 


\section{Conclusion}

Amoderate size shock tube $(7 \mathrm{~m})$ has been installed in the Mechanical Engineering Department at Indian Institute of Technology Guwahati. It has been calibrated successfully by measuring and estimating shock tube parameters through experiments and analytical calculations with average deviation within $\pm 10 \%$. Most of the components and instrumentations of this shock tube are indigenously designed and fabricated. In the areas of aerospace science and technologies, the attention can be focused to simulate hypersonic flows experimentally through design of suitable convergent-divergent nozzles. The future scope of this facility development is also inclined towards many interesting mechanical applications in the areas of impact assessment on structures and shock assisted deformation studies for metals and composites, chemical kinetics, ignition delay measurements for potential bio-fuels.

\section{Acknowledgements}

None.

\section{Conflict of interest}

Author declares that there is no conflict of interest.

\section{References}

1. Van Dyke MD. The supersonic blunt body problem-Review and extension. Journal of Aerospace Sciences. 1958;25(8):485-496.

2. Charles EW, Merle RW. Heat transfer to slender cones in hypersonic air flow including effects of the yaw and nose bluntness. Journal of Aerospace Sciences. 1962;29(7):761-774.

3. Modarress D, Azzazy M. Modern experimental techniques for high speed flow measurements. 26th AIAA aerospace conference. Reno, NV, USA; 1988.420 p.

4. Lu FK, Wilson DR. Survey of short duration, hypersonic and hypervelocity facilities. 25th Plasmadynamics and Lasers Conference. Colorado, USA; 1994. p. 94-2491.

5. Doolan CJ, Morgan RG. A two-stage free-piston driver. Shock Waves. 1999;9(4):239-248.
6. Itoh K, Komuro T, Sato K, et al. Hypersonic aerodynamic research of HOPE using high enthalpy shock tunnel, 10th AIAA. 2001. p. 1-1824.

7. Stalker RJ, Paull A, Mee DJ, et al. Scramjets and shock tunnels-The Queeensland experience. Progress in Aerospace Sciences. 2005;41:471-513.

8. Reddy NM. Development of a hypersonic shock tunnel. Proceedings of Indian Academy Sciences. 1978;1(1):73-92.

9. Reddy NM, Nagashetty K, Jagadeesh G, et al. Review of hypersonic research investigations in the IISc shock tunnel (HST1). Proceedings of Indian Academy Science. 1996;21(6):741-773.

10. Vidal RJ. Model instrumentation techniques for heat transfer and force measurements in a hypersonic shock tunnel. Cornell Aeronautical Laboratory Report, WADC TN, Buffalo, N.Y. New York. USA; 1956. p. 56-315.

11. Schultz DL, Jones TV. Heat transfer measurements in short-duration hypersonic facilities. AGARDograph-AG-165, Advisory Group For Aerospace Research And Development Paris. France; 1973. 155 p.

12. Joshi MV, Reddy NM. Aerodynamic force measurements over missile configurations in IISc shock tunnel at $\mathrm{M}_{\infty}=5.5$. Experiments in Fluids. 1986;4(6):338-340.

13. Raju C, Reddy NM. Aerodynamic force measurements over missile configurations in IISc shock tunnel at $\mathrm{M}_{\infty}=3.85$ and 9.15 , Experiments in Fluids. 1990;10(2):175-177.

14. Sahoo N, Kulkarni V, Saravanan S, et al. Film cooling effectiveness on a large angle blunt cone flying at hypersonic speed, Physics of Fluids. 2005;17(3):1-11.

15. Sahoo N, Saravanan S, Jagadeesh G, et al. Simultaneous measurement of aerodynamic and heat transfer data for large angle blunt cones in hypersonic shock tunnel, Academy Proceedings in Engineering Sciences, $S A$ DHANA. 2006;31(5):557-581.

16. Sahoo N. Simultaneous measurement of aerodynamic force and convective surface heat transfer rates for large angle blunt cones in hypersonic shock tunnel. PhD Dissertation, Indian Institute of Science, Bangalore, India; 2003.

17. Gaydon AG, Hurle IR. The Shock Tube in High-Temperature Chemical Physics. New York: The Reinhold Publishing Corporation, London, UK; 1963. 